GANIT J. Bangladesh Math. Soc. (ISSN 1606-3694) 37 (2017) 93-98

\title{
A NOTE ON $\alpha$-MULTIPLIERS OF PRIME RINGS
}

\author{
Kyung Ho Kim \\ Department of Mathematics, Korea National University of Transportation \\ Chungju 380-702, Korea \\ E-mail: ghkim@ut.ac.kr
}

Received 09.04.2017 Accepted 30.07.2017

\begin{abstract}
In this paper, we investigate the commutativity of prime rings admitting $\boldsymbol{\alpha}$-multipliers of $\mathrm{R}$ satisfying certain identities and some related results have also been discussed.
\end{abstract}

Keywords: $\alpha$-multiplier, prime, 2-torsion free, commutative, automorphism.

\section{Introduction}

An additive mapping $d: R \rightarrow R$ is called a derivation if $d(x y)=d(x) y+y d(x)$ holds for all $\mathrm{x}, \mathrm{y} \in \mathrm{R}$. Over the last few decades, several authors have investigated the relationship between the commutativity of the ring $\mathrm{R}$ and certain specific types of derivations of $\mathrm{R}$. The first result in this direction is due to E. C. Posner [9] who proved that if a ring $\mathrm{R}$ admits a nonzero derivation $\mathrm{d}$ such that $[\mathrm{d}(\mathrm{x}), \mathrm{x}] \in \mathrm{Z}(\mathrm{R})$ for all $\mathrm{x} \in \mathrm{R}$, then $\mathrm{R}$ is commutative. This result was subsequently, refined and extended by a number of authors. In [7], Bresar and Vuckman showed that a prime ring must be commutative if it admits a nonzero left derivation. Recently, many authors have obtained commutativity theorems for prime and semiprime rings admitting derivation, generalized derivation. Many considerable works have been done on left (right) multipliers in prime and semiprime rings during the last couple of decades ([10-12]). In this paper, we investigate the commutativity of prime rings admitting $\alpha$-multipliers of $\mathrm{R}$ satisfying certain identities and some related results have also been discussed.

\section{Preliminaries}

Throughout $\mathrm{R}$ will represent an associative ring with center $\mathrm{Z}(\mathrm{R})$. For all $\mathrm{x}, \mathrm{y} \in \mathrm{R}$, as a usual commutator, we shall write $[x, y]=x y-y x$, and $x \circ y=x y+y x$. Also, we make use of the following two basic identities without any specific mention:

$$
\begin{aligned}
& x \circ(y z)=(x \circ y) z-y[x, z]=y(x \circ z)+[x, y] z \\
& (x y) \circ z=x(y \circ z)-[x, z] y=(x \circ z) y+x[y, z] \\
& {[x y, z]=x[y, z]+[x, z] y \text { and }[x, y z]=y[x, z]+[x, y] z .}
\end{aligned}
$$

Let $R$ is a ring. An additive mapping $F: R \rightarrow R$ is called a left multiplier if $F(x y)=F(x) y$ holds for every $\mathrm{x}, \mathrm{y} \in \mathrm{R}$. Similarly, an additive mapping $\mathrm{F}: \mathrm{R} \rightarrow \mathrm{R}$ is called a right multiplier if $\mathrm{F}(\mathrm{xy})=$ 
$\mathrm{xF}(\mathrm{y})$ holds for every $\mathrm{x}, \mathrm{y} \in \mathrm{R}$. If $\mathrm{F}$ is both a left and a right multiplier of $\mathrm{R}$, then it is called a multiplier of R. An additive

mapping $\mathrm{F}: \mathrm{R} \rightarrow \mathrm{R}$ is called a generalized derivation if there exists a derivation $\mathrm{d}$ : $\mathrm{R} \rightarrow \mathrm{R}$ such that $F(x y)=F(x) y+x d(y)$ holds for all $x, y \in R$, and $d$ is called the associated derivation of $F$. Obviously, a generalized derivation with $d=0$ covers the concept of left multipliers. It is easy to see that $F: R \rightarrow R$ is a generalized derivation if and only if $F$ is of the form $F=d+H$, where $d$ is a derivation and $\mathrm{H}$ is a left multiplier. An additive mapping $\mathrm{F}: \mathrm{R} \rightarrow \mathrm{R}$ is called an $\alpha$-multiplier if $\mathrm{F}(\mathrm{xy})=\mathrm{F}(\mathrm{x}) \alpha(\mathrm{y})=\alpha(\mathrm{x}) \mathrm{F}(\mathrm{y})$ holds for every $\mathrm{x}, \mathrm{y} \in \mathrm{R}$, where $\alpha: \mathrm{R} \rightarrow \mathrm{R}$ is a mapping.

Lemma 2.1 Let $\mathrm{R}$ be a prime ring. If $\mathrm{F}$ is a nonzero $\alpha$-multiplier of $\mathrm{R}$ associated with an onto map $\alpha$, then $F(x) \in Z(R)$ for all $x \in Z(R)$.

Proof. Let $\mathrm{z} \in \mathrm{Z}(\mathrm{R})$. By definition of $\mathrm{F}$, we have

$$
\mathrm{F}(\mathrm{xz})=\alpha(\mathrm{x}) \mathrm{F}(\mathrm{z})=\mathrm{F}(\mathrm{z}) \alpha(\mathrm{x})=\mathrm{F}(\mathrm{zx})
$$

for every $\mathrm{x} \in \mathrm{R}$. Since $\alpha$ is onto, we have $\mathrm{xF}(\mathrm{z})=\mathrm{F}(\mathrm{z}) \mathrm{x}$ for all $\mathrm{x} \in \mathrm{R}$, and so $\mathrm{F}(\mathrm{z}) \in \mathrm{Z}(\mathrm{R})$.

Lemma 2.2 ([3]) Let $R$ be a prime ring. If $z \in Z(R)-\{0\}$ and $x z \in Z(R)$, then $x \in Z(R)$.

\section{3. $\alpha$-multipliers of prime rings}

Theorem 3.1 Let $\mathrm{R}$ be a prime ring and let $\alpha$ be an automorphism of $\mathrm{R}$. If $\mathrm{F}: \mathrm{R} \rightarrow \mathrm{R}$ is a nonzero $\alpha$-multiplier of $\mathrm{R}$ and $\mathrm{F}(\mathrm{R}) \subseteq \mathrm{Z}(\mathrm{R})$, then $\mathrm{R}$ is commutative.

Proof. Assume that $F(x) \in Z(R)$ for all $x \in R$. Then we have

$$
\mathrm{F}(\mathrm{xy}) \alpha(\mathrm{t})=\alpha(\mathrm{t}) \mathrm{F}(\mathrm{xy}), \forall \mathrm{x}, \mathrm{y}, \mathrm{t} \in \mathrm{R},
$$

which implies that $\mathrm{F}(\mathrm{x}) \alpha(\mathrm{y}) \alpha(\mathrm{t})=\alpha(\mathrm{t}) \mathrm{F}(\mathrm{x}) \alpha(\mathrm{y})$ for all $\mathrm{x}, \mathrm{y}, \mathrm{t} \in \mathrm{R}$. Hence

$$
\mathrm{F}(\mathrm{x}) \alpha(\mathrm{y}) \alpha(\mathrm{t})=\mathrm{F}(\mathrm{x}) \alpha(\mathrm{t}) \alpha(\mathrm{y}) \forall \mathrm{x}, \mathrm{y}, \mathrm{t} \in \mathrm{R},
$$

That is, $F(x)[\alpha(y), \alpha(t)]=0$ for all $x, y, t \in R$. Replacing $y$ by $y x$ in this relation, we have $\mathrm{F}(\mathrm{x}) \alpha(\mathrm{y})[\alpha(\mathrm{x}), \alpha(\mathrm{t})]=0$ for all $\mathrm{x}, \mathrm{y}, \mathrm{t} \in \mathrm{R}$. This implies that $\mathrm{F}(\mathrm{x}) \mathrm{R}[\alpha(\mathrm{x}), \alpha(\mathrm{t})]=\{0\}$ for all $x, t \in R$. Since $R$ is prime, we have $F(x)=0$ or $[\alpha(x), \alpha(t)]=0$ for all $x, t \in R$. Let $K=$ $\{\mathrm{x} \in \mathrm{R} \mid \mathrm{F}(\mathrm{x})=0\}$ and $\mathrm{L}=\{\mathrm{x} \in \mathrm{R} \mid[\alpha(\mathrm{x}), \alpha(\mathrm{y})]=0, \forall \mathrm{y} \in \mathrm{R}\}$. Then $\mathrm{K}$ and $\mathrm{L}$ are both additive subgroups and $K \cup L=R$, but $(R,+)$ is not union of two its proper subgroups, which implies that either $\mathrm{K}=\mathrm{R}$ or $\mathrm{L}=\mathrm{R}$. In the former case, we have $\mathrm{F}=0$, contradiction, and so $\mathrm{L}=\mathrm{R}$, that is, $[\alpha(x), \alpha(y)]=0$ for all $x, y \in R$. Since $\alpha$ is onto, we have $[x, y]=0$ for all $x, y \in R$, which implies that $\mathrm{R}$ is commutative.

Theorem 3.2 Let $\mathrm{R}$ be a prime ring and let $\alpha$ be an automorphism of $\mathrm{R}$ and $\mathrm{F}: \mathrm{R} \rightarrow \mathrm{R}$ be an $\alpha$ multiplier of $R$. If $F(x y)=F(x) F(y)$ for all $x, y \in R$ and $F(x) \neq x$ for all $x \in R$, then $F=0$.

Proof. By hypothesis, we have 


$$
\mathrm{F}(\mathrm{xy})=\mathrm{F}(\mathrm{x}) \alpha(\mathrm{y})=\mathrm{F}(\mathrm{x}) \mathrm{F}(\mathrm{y}), \forall \mathrm{x} \in \mathrm{R} .
$$

Replacing $\mathrm{x}$ by $\mathrm{xw}$ in (3), we have $\mathrm{F}(\mathrm{xw}) \alpha(\mathrm{y})=\mathrm{F}(\mathrm{xw}) \mathrm{F}(\mathrm{y})$, that is, $\mathrm{F}(\mathrm{x}) \alpha(\mathrm{w}) \alpha(\mathrm{y})=$ $\mathrm{F}(\mathrm{x}) \alpha(\mathrm{w}) \mathrm{F}(\mathrm{y})$ for all $\mathrm{x}, \mathrm{y}, \mathrm{w} \in \mathrm{R}$. This implies that $\mathrm{F}(\mathrm{x}) \alpha(\mathrm{w})(\alpha(\mathrm{y})-\mathrm{F}(\mathrm{y}))=0$ for all $\mathrm{x}, \mathrm{y}, \mathrm{w} \in$ R. Taking $\alpha^{-1}(\mathrm{w})$ instead of $w$ in this relation, we have $F(x) w(\alpha(y)-F(y))=0$, and so $F(x) R(\alpha(y)-F(y))=\{0\}$. Since $\alpha$ is onto, we have $F(x) R(y-F(y))=\{0\}$. Since $R$ is prime, we have $F(x)=0$ for all $x \in R$ or $y-F(y)=0$ for all $y \in R$. But $F(x) \neq x$ for all $x \in R$, and so $\mathrm{F}(\mathrm{x})=0$ for all $\mathrm{x} \in \mathrm{R}$.

Theorem 3.3 Let $\mathrm{R}$ be a prime ring and let $\alpha$ be an onto mapping on $\mathrm{R}$. If $\mathrm{F}$ is a nonzero $\alpha$ multiplier of $\mathrm{R}$ such that $\mathrm{F}([\mathrm{x}, \mathrm{y}]) \in \mathrm{Z}(\mathrm{R})$ for all $\mathrm{x}, \mathrm{y} \in \mathrm{R}$ and $\mathrm{F}(\mathrm{Z}(\mathrm{R})) \neq 0$, then $\mathrm{R}$ is commutative.

Proof. By hypothesis, we have

$$
\mathrm{F}([\mathrm{x}, \mathrm{y}]) \in \mathrm{Z}(\mathrm{R}), \forall \mathrm{x}, \mathrm{y} \in \mathrm{R} \text {. }
$$

Since $F(Z(R)) \neq 0$, there exists $z \in Z(R)$ such that $F(z) \neq 0$. Thus $F(z) \in Z(R)$ by Lemma 2.1. Replacing $\mathrm{x}$ by zx in (4),

$$
\mathrm{F}(\mathrm{z}[\mathrm{x}, \mathrm{y}]) \in \mathrm{Z}(\mathrm{R}), \forall \mathrm{x}, \mathrm{y}, \mathrm{z} \in \mathrm{R},
$$

which implies that $F(z) \alpha([x, y]) \in Z(R)$ for all $x, y, z \in R$. Since $R$ is prime and $F(z) \neq 0$, we have $\alpha([x, y]) \in Z(R)$ for all $x, y \in R$. Using the fact that $\alpha$ is onto, we get $[x, y] \in Z(R)$ for all $\mathrm{x}, \mathrm{y} \in \mathrm{R}$. This implies that

$$
[\mathrm{r},[\mathrm{x}, \mathrm{y}]]=0, \forall \mathrm{x}, \mathrm{y}, \mathrm{r} \in \mathrm{R} \text {. }
$$

Taking yx instead of $y$ in the relation (6), we have $[y, x][r, x]=0$ for all $x, y, r \in R$.

Again, replacing $r$ by rs in the last relation, we have $[y, x] R[s, x]=\{0\}$ for all $x, y, s \in R$. Since $R$ is prime, we have either $[\mathrm{y}, \mathrm{x}]=0$ or $[\mathrm{s}, \mathrm{x}]=0$ for all $\mathrm{x}, \mathrm{y} \in \mathrm{R}$. Let $\mathrm{K}=\{\mathrm{x} \in \mathrm{R} \mid[\mathrm{y}, \mathrm{x}]=0\}$ and $\mathrm{L}=$ $\{\mathrm{x} \mid[\mathrm{s}, \mathrm{x}]=0\}$. Then $\mathrm{K}$ and $\mathrm{L}$ are both additive subgroups and $\mathrm{K} \cup \mathrm{L}=\mathrm{R}$, but $(\mathrm{R},+)$ is not union of two its proper subgroups, which implies that either $\mathrm{K}=\mathrm{R}$ or $\mathrm{L}=\mathrm{R}$. That is, In both cases, $\mathrm{R}$ is commutative.

Theorem 3.4 Let $\mathrm{R}$ be a prime ring and let $\alpha$ be an onto map on $\mathrm{R}$. If $\mathrm{F}$ is a nonzero $\alpha$-multiplier of $R$ such that $F(x \circ y) \in Z(R)$ for all $x, y \in R$ and $F(Z(R)) \neq 0$, then $R$ is commutative.

Proof. By hypothesis, we have

$$
\mathrm{F}(\mathrm{x} \circ \mathrm{y}) \in \mathrm{Z}(\mathrm{R}), \forall \mathrm{x}, \mathrm{y} \in \mathrm{R} \text {. }
$$

Since $F(Z(R)) \neq 0$, there exists $z \in Z(R)$ such that $F(z) \neq 0$. Thus $F(z) \in Z(R)$ by Lemma 2.1 . Replacing $\mathrm{x}$ by zx in (7), we have

$$
\mathrm{F}(\mathrm{z}(\mathrm{x} \circ \mathrm{y})) \in \mathrm{Z}(\mathrm{R}), \forall \mathrm{x}, \mathrm{y}, \mathrm{z} \in \mathrm{R},
$$


which implies that $F(z) \alpha(x \circ y) \in Z(R)$ for all $x, y, z \in R$. Since $R$ is prime and $F(z) \neq 0$, we have $\alpha(x \circ y) \in Z(R)$ for all $x, y \in R$. Using the fact that $\alpha$ is onto, we get $x \circ y \in Z(R)$ for all $\mathrm{x}, \mathrm{y} \in \mathrm{R}$. Replacing $\mathrm{x}$ by $\mathrm{xz}$ in the last relation and using the fact that $\mathrm{yx}=-\mathrm{xy}$, we obtain $x[z, y]=0$ for all $x, y, z \in R$. That is, $R[z, y]=\{0\}$. This implies that $x R[z, y]=\{0\}$ for $0 \neq x \in R$. Since $\mathrm{R}$ is prime, we have $[\mathrm{z}, \mathrm{y}]=0$ for all $\mathrm{y}, \mathrm{z} \in \mathrm{R}$, which means that $\mathrm{R}$ is commutative.

Theorem 3.5 Let $\mathrm{R}$ be a prime ring and let $\alpha$ be an onto map on $\mathrm{R}$. If $\mathrm{F}$ is a nonzero $\alpha$-multiplier of $R$ such that $x \circ F(y) \in Z(R)$ for all $x, y \in R$ and $F(Z(R)) \neq 0$, then $R$ is commutative.

Proof. By hypothesis, we have

$$
\mathrm{x} \circ \mathrm{F}(\mathrm{y}) \in \mathrm{Z}(\mathrm{R}), \forall \mathrm{x}, \mathrm{y} \in \mathrm{R} .
$$

Since $F(Z(R)) \neq 0$, there exists $z \in Z(R)$ such that $F(z) \neq 0$. Thus $F(z) \in Z(R)$ by Lemma 2.1 . Replacing y by zy in (9), we have $F(z)(x \circ \alpha(y)) \in Z(R)$ for all $x, y \in R$. Since $R$ is prime and $\mathrm{F}(\mathrm{z}) \neq 0$, we have $\mathrm{x} \circ \alpha(\mathrm{y}) \in \mathrm{Z}(\mathrm{R})$ for all $\mathrm{x}, \mathrm{y} \in \mathrm{R}$. Using the fact that $\alpha$ is onto, we get $\mathrm{x} \circ \mathrm{y} \in$ $Z(R)$ for all $x, y \in R$. Using the same argument of the last part of proof of Theorem 3.4, we get the required result.

Theorem 3.6 Let $\mathrm{R}$ be a prime ring and let $\alpha$ be an onto map on $\mathrm{R}$. If $\mathrm{F}$ is a nonzero $\alpha$-multiplier of $R$ such that $[x, F(y)] \in Z(R)$ for all $x, y \in R$ and $F(Z(R)) \neq 0$, then $R$ is commutative.

Proof. By hypothesis, we have

$$
[\mathrm{x}, \mathrm{F}(\mathrm{y})] \in \mathrm{Z}(\mathrm{R}), \forall \mathrm{x}, \mathrm{y} \in \mathrm{R} .
$$

Since $F(Z(R)) \neq 0$, there exists $z \in Z(R)$ such that $F(z) \neq 0$. Thus $F(z) \in Z(R)$ by Lemma 2.1 Replacing y by zy in (10), we have $F(z)[x, \alpha(y)]+[x, F(z)] \alpha(y) \in Z(R)$ for all $x, y \in R$, which implies that $F(z)[x, \alpha(y)] \in Z(R)$ for all $x, y \in R$. Since $R$ is prime and $F(z) \neq 0$, we have $[x, \alpha(y)] \in Z(R)$ for all $x, y \in R$. Using the fact that $\alpha$ is onto, we get $[x, y] \in Z(R)$ for all $\mathrm{x}, \mathrm{y} \in \mathrm{R}$. Using the same argument of the last part of proof of Theorem 3.3, we get the required result.

Theorem 3.7 Let $\mathrm{R}$ be a prime ring and let $\alpha$ be an onto map on $\mathrm{R}$. If $\mathrm{F}$ is a nonzero $\alpha$-multiplier of $R$ such that $[F(x), F(y)] \in Z(R)$ for all $x, y \in R$ and $F(Z(R)) \neq 0$, then $R$ is commutative.

Proof. By hypothesis, we have

$$
[\mathrm{F}(\mathrm{x}), \mathrm{F}(\mathrm{y})] \in \mathrm{Z}(\mathrm{R}), \forall \mathrm{x}, \mathrm{y} \in \mathrm{R} .
$$

Since $F(Z(R)) \neq 0$, there exists $z \in Z(R)$ such that $F(z) \neq 0$. Thus $F(z) \in Z(R)$ by Lemma 2.1. Replacing y by zy in (11), we have $F(z)[F(x), \alpha(y)]+[F(x), F(z)] \alpha(y) \in Z(R)$ for all $x, y \in R$, which implies that $F(z)[F(x), \alpha(y)] \in Z(R)$ for all $x, y \in R$. Since $R$ is prime and $F(z) \neq 0$, we have $[F(x), \alpha(y)] \in Z(R)$ for all $x, y \in R$. Using the fact that $\alpha$ is onto, we get $[F(x), y] \in Z(R)$ for all $x, y \in R$. By the Theorem 3.6, we get the required result. 
Theorem 3.8 Let $\mathrm{R}$ be a prime ring and let $\alpha$ be an onto map on $\mathrm{R}$. If $\mathrm{F}$ is a nonzero $\alpha$-multiplier of $R$ such that $F(x) \circ F(y) \in Z(R)$ for all $x, y \in R$ and $F(Z(R)) \neq 0$, then $R$ is commutative.

Proof. By hypothesis, we have

$$
\mathrm{F}(\mathrm{x}) \circ \mathrm{F}(\mathrm{y}) \in \mathrm{Z}(\mathrm{R}), \forall \mathrm{x}, \mathrm{y} \in \mathrm{R} \text {. }
$$

Since $F(Z(R)) \neq 0$, there exists $z \in Z(R)$ such that $F(z) \neq 0$. Thus $F(z) \in Z(R)$ by Lemma 2.1. Replacing y by zy in (12), we have $F(z)(F(x) \circ \alpha(y))+[F(x), F(z)] \alpha(y) \in Z(R)$ for all $x, y \in$ $R$, which implies that $F(z)(F(x) \circ \alpha(y)) \in Z(R)$ for all $x, y \in R$. Since $R$ is prime and $F(z) \neq 0$, we have $\mathrm{F}(\mathrm{x}) \circ \alpha(\mathrm{y}) \in \mathrm{Z}(\mathrm{R})$

for all $x, y \in R$. Using the fact that $\alpha$ is onto, we get $F(x) \circ y \in Z(R)$ for all $x, y \in R$. By the Theorem 3.5, we get the required result.

Theorem 3.9 Let $\mathrm{R}$ be a prime ring and let $\alpha$ be an onto map on $\mathrm{R}$. If $\mathrm{F}$ is a nonzero $\alpha$-multiplier of $R$ such that $[F(x), y]-[x, y] \in Z(R)$ for all $x, y \in R$ and $F(Z(R)) \neq 0$ and $F(x) \neq x$ for all $x \in$ $\mathrm{R}$, then $\mathrm{R}$ is commutative.

Proof. By hypothesis, we have

$$
[F(x), y]-[x, y] \in Z(R), \forall x, y \in R .
$$

Since $F(Z(R)) \neq 0$, there exists $z \in Z(R)$ such that $F(z) \neq 0$. Thus $F(z) \in Z(R)$ by Lemma 2.1. Replacing $x$ by $z x$ in (13), we have $F(z)[(x), y]-z[x, y] \in Z(R)$ for all $x, y \in R$. Since $\alpha$ is onto, we have

$$
\mathrm{F}(\mathrm{z})[\mathrm{x}, \mathrm{y}]-\mathrm{z}[\mathrm{x}, \mathrm{y}]=(\mathrm{F}(\mathrm{z})-\mathrm{z})[\mathrm{x}, \mathrm{y}] \in \mathrm{Z}(\mathrm{R}), \forall \mathrm{x}, \mathrm{y} \in \mathrm{R} .
$$

Since $R$ is prime and $F(z)-z \neq 0$, we have $[x, y] \in Z(R)$ for all $x, y \in R$. This implies that $R$ is commutative.

Theorem 3.10 Let $\mathrm{R}$ be a prime ring and let $\alpha$ be an onto map on $\mathrm{R}$. If $\mathrm{F}$ is a nonzero $\alpha$-multiplier of $R$ such that $F(x) \circ y-(x \circ y) \in Z(R)$ for all $x, y \in R$ and $F(Z(R)) \neq 0$ and $F(x) \neq$ $\mathrm{x}$ for all $\mathrm{x} \in \mathrm{R}$, then $\mathrm{R}$ is commutative.

Proof. By hypothesis, we have

$$
\mathrm{F}(\mathrm{x}) \circ \mathrm{y}-(\mathrm{x} \circ \mathrm{y}) \in \mathrm{Z}(\mathrm{R}), \forall \mathrm{x}, \mathrm{y} \in \mathrm{R} .
$$

Since $F(Z(R)) \neq 0$, there exists $z \in Z(R)$ such that $F(z) \neq 0$. Thus $F(z) \in Z(R)$ by Lemma 2.1 . Replacing $x$ by $z x$ in $(15)$, we have $F(z)(\alpha(x) \circ y)-z(x \circ y) \in Z(R)$ for all $x, y, z \in R$. Since $\alpha$ is onto, we have

$$
\mathrm{F}(\mathrm{z})(\alpha(\mathrm{y}) \circ \mathrm{y})-\mathrm{z}(\mathrm{x} \circ \mathrm{y})=(\mathrm{F}(\mathrm{z})-\mathrm{z})(\mathrm{x} \circ \mathrm{y}) \in \mathrm{Z}(\mathrm{R}), \forall \mathrm{x}, \mathrm{y} \in \mathrm{R} .
$$

Since $R$ is prime and $F(z)-z \neq 0$, we have $x \circ y \in Z(R)$ for all $x, y \in R$. Using the same argument of the last part of proof of Theorem 3.4 we get the required result. 
Theorem 3.11 Let $\mathrm{R}$ be a prime ring and let $\alpha$ be an onto map on $\mathrm{R}$. If $\mathrm{F}$ is a nonzero $\alpha$-multiplier of $R$ such that $[F(x), F(y)]-[x, y] \in Z(R)$ for all $x, y \in R$ and $F(Z(R)) \neq 0$, then $R$ is commutative.

Proof. By hypothesis, we have

$$
[F(x), F(y)]-[x, y] \in Z(R), \forall x, y \in R .
$$

Since $F(Z(R)) \neq 0$, there exists $z \in Z(R)$ such that $F(z) \neq 0$. Thus $F(z) \in Z(R)$ by Lemma 2.1 . Replacing $x$ by $z x$ in (17), we have $F(z)[\alpha(x), F(y)]-z[x, y] \in Z(R)$ for all $x, y, z \in R$. Taking $y$ instead of $\mathrm{x}$ in the last relation, we have

$$
\mathrm{F}(\mathrm{z})[\alpha(\mathrm{y}), \mathrm{F}(\mathrm{y}) \in \mathrm{Z}(\mathrm{R}), \forall \mathrm{y}, \mathrm{z} \in \mathrm{R} .
$$

Since $R$ is prime and $F(z) \neq 0$, we have $[\alpha(y), F(y)] \in Z(R)$ for all $y \in R$. Since $\alpha$ is onto, we have $[y, F(y)] \in Z(R)$ for all $y \in R$ By Theorem 3.6, we get the required result.

\section{REFERENCES}

[1] M. Ashraf and S. Ali, On $(\sigma, \tau)$-derivations of prime rings, Sarajevo J. Math 16 (2008), 23-30.

[2] M. Ashraf and S. Ali, On left multipliers and the commutativity of rings, DEMONSTRATIO MATHEMATICA 42 (4) (2008), 763-771.

[3] H. E. Bell, On derivations in near-rings II, Near-rings, Near-_eld and K-loops, Hamburg, 1995), 191 197 Math. Appl., 426, Kluwer Acad.publ., Dordrecht, 1997.

[4] H. E. Bell and M. N. Daif, On commutativity and strong commutativity preserving maps, Canad. Math, Bull 37 (1994), 443-447.

[5] M. Bresar, On a generalization of the notion of centralizing mappings, Proc. Amer. Math. Soc, 114 (1992), 641-649.

[6] M. Bresar, On the distance the composition of two derivation to the generalized derivations, Glasgow Math. J. 33 (1991), 89-93.

[7] M. Bresar and J. Vukman, On left derivations and related mappings, Proc. Amer. Math. Soc, 110 (1990), 7-16.

[8] O. Golbasi, Some properties of prime near-rings with $(\sigma, \tau)$-derivation, Siberian Mathematical Journal, 46 (2005), 270-275.

[9] E. C. Posner, Derivations in prime rings, Proc. Amer. Math. Soc, 8 (1957), 1093-1100.

[10] J. Vukman, Centralizer on semiprime rings, Comment. Math. Univ. Carolinae, 42 (2001), 237-245.

[11] J. Vukman, Identity related to centralizer in semiprime rings, Comment. Math. Univ. Carolinae, 40 (1999), 447-456.

[12] B. Zalar, On centralizer of semiprime rings, Comment. Math. Univ. Carolinae, 32 (1991), 609-614. 